\title{
Development of a repeated measures affective change blindness task
}

\author{
Antony C. Moss • Ian P. Albery • Dinkar Sharma
}

Published online: 17 March 2011

(C) Psychonomic Society, Inc. 2011

\begin{abstract}
Flicker-induced change blindness paradigms have been used to explore attentional biases for a range of concern-related cues. However, previous studies have had limitations related to concerns about carryover effects in repeated measures designs, as well as problems with response modalities. The present article develops a repeated measures paradigm utilising nonverbal responses and explores the implications of these design modifications for the reliability and validity of the paradigm. Affective stimuli were presented to participants, and the results suggest that the modified paradigm is a useful tool for assessing attentional bias. A number of recommendations for the future use of this methodology are made.
\end{abstract}

Keywords Flicker-induced change blindness · Attentional bias - Affective stimuli · Methodology

Sensorial perception, for example, certainly occurs with greater or less accuracy according to the degree of interest; it is constantly given other directions by the change of external stimuli and by ideas.

-Hermann Ebbinghaus (1913), Memory: A Contri-

bution to Experimental Psychology

The study of change blindness and inattentional blindness has demonstrated that what one is looking at is not always

A. C. Moss $(\bowtie) \cdot$ I. P. Albery

Department of Psychology, London South Bank University,

London, UK

e-mail: mossac@1sbu.ac.uk

D. Sharma

School of Psychology, University of Kent,

Canterbury, Kent, UK necessarily seen. In typical change blindness research, participants are presented with a rapidly flickering scene within which a change is occurring. Between the "changed" and "not changed" images, a mask is inserted (e.g., a screen of white noise) that increases the difficulty participants experience in detecting often very large changes in the image. For example, the engine from an aeroplane may disappear and reappear, or the horizon may shift position by a few degrees (see Rensink, 2002, for a review). The most surprising finding from much of this research is that when such changes occur, participants may often take upwards of $10 \mathrm{~s}$ to notice what is changing. Simons and Chabris (1999) extended the basic paradigm beyond still images to show that inattentional blindness can occur in moving images, too. They found that as few as $8 \%$ of participants noticed a gorilla that stopped in the middle of the screen, beat his chest, and walked out of shot, while the participants were busy counting the number of passes made by basketball players onscreen. As might be expected, detection rates were negatively affected by making the gorilla transparent and by giving participants a more difficult task to perform whilst watching the video.

Change blindness is thought to occur because individuals are not able to detect motion cues in these artificial “flickering" paradigms (Rensink, O'Regan, \& Clark, 1997; Simons \& Rensink, 2005). Rensink et al. (1997) suggest that perception of a change requires direct attention to be paid to and maintained on the changing object for detection to occur. As a result, when the changing items in a flicker task hold intrinsic interest for the participants, they are likely to detect the change more quickly.

Whilst change blindness paradigms have been primarily applied in perception research to study the nature of visual attention, more recently they have been adapted to study attentional biases towards concern-related cues. In one study, American football players were shown images of 
footballers on a pitch standing in tactical formations. When a change occurred that was relevant to the strategy of the supposed game (e.g., a defender disappeared and reappeared), experienced football players were significantly faster than less experienced players at detecting the change (Werner \& Thies, 2000). Similarly, B. T. Jones, Macphee, Broomfield, Jones, and Espie (2005) found significantly greater attentional biases in a group of poor sleepers, as compared to good and moderate sleepers, towards bedroom-related stimuli. In a series of articles, B. T. Jones and colleagues used a flicker paradigm to measure attentional biases amongst problem, nondependent alcohol and cannabis users, using images containing both substance-neutral and substance-related (alcohol or cannabis) objects (Beare, Sturt, Bruce, \& Jones, 2007; B. C. Jones, Jones, Blundell, \& Bruce, 2002; B. T. Jones, Bruce, Livingstone, \& Reed, 2006; B. T. Jones, Jones, Smith, \& Copley, 2003). During each trial in these studies, a change would occur to either one or both objects, with participants who reported higher levels of substance use being significantly more likely to report seeing the substance-related change first and/or detecting this change more quickly than those who detected the substance-neutral change.

The flicker-induced change blindness paradigm is clearly a useful tool for the assessment of attentional biases, and it adds to the growing number of tools available to researchers interested in this phenomenon. In addition, the paradigm also allows researchers to present multiple stimuli to participants, not just on the same screen, as would be the case in a dotprobe-type task (e.g., McCabe, Gotlib, \& Martin, 2000), but within the same context. That is, rather than presenting participants with a picture of a cigarette alongside a separate picture of a pen, in this paradigm one might present instead a picture of two people in some social interaction, one of whom is holding a pen, the other a cigarette. This allows for far richer and more complex stimuli to be presented to participants, more closely matching the environments that researchers may wish to extrapolate their findings to. However, there are some limitations to the current design of this paradigm.

Previous implementations of the flicker paradigm for use as a measure of attentional bias have typically used between-participants designs, with participants providing one response to either a neutral or a concern-related change and giving a verbal response to identify the object that has changed. B. T. Jones et al. (2003) suggested that the semantic nature of a stimulus becomes active in memory when responses are given verbally, leading to carryover effects on subsequent trials. The assumption appears to be that experiments utilising multiple measures of change blindness within participants is problematic to the extent that such carryover effects can not be ruled out. However, this assumption has not been assessed empirically. In addition, the use of multiple changes per trial incorporating a verbal response modality could theoretically lead to another form of response bias not previously considered. Given that participants in these previous studies were asked to (1) press a button when they saw a change occurring and (2) verbally report the change "seen," this does not discount the possibility that participants may have identified both changes during the time taken to initiate a motor response and halt the flicker trial. As a result, participants' verbal responses to the change identified may not necessarily reflect the same change that initiated the button response.

Here we develop the flicker paradigm for assessing attentional biases used in previous research, using a repeated measures design with nonverbal responses. We investigated the possibility that repeated trials, across multiple trial types, would produce some form of response bias. While previous researchers have suggested that this could be a problem in repeated measures designs when assessing attentional biases using this paradigm, there has been no evidence to demonstrate whether or not these concerns are justified.

In the present study, affective stimuli were presented in visual images, with a single change occurring during each trial to either a positive, a neutral, or a negative object within each image, to provide attentional bias measures for different types of affective change. This in itself is an important departure from other paradigms that are used for assessing attentional bias; it is most common in this domain to juxtapose pairs of stimuli in order to identify which distracts a participant's attention the most. In real life, it is hard to imagine a similar situation in which only two possible stimuli are competing for one's attention. This is a significant advantage of the flicker paradigm: that the richness of stimuli that one might present can far more readily equate to real-world situations.

It was not clear at the outset of this study what differences in response latencies towards positive, neutral, and negative objects would be apparent. However, previous research using other paradigms has suggested that a negativity bias would be likely, due to the threat relatedness of negative objects (e.g., Fox, Russo, \& Dutton, 2002; Ito, Larsen, Smith, \& Cacioppo, 1998). For example, Segerstrom (2001) identified a cognitive bias for affective stimuli as a function of levels of optimism, such that decreased levels of optimism were associated with a greater bias towards negative stimuli in an emotional Stroop task. Also using an emotional Stroop task, Drobes, Elibero, and Evans (2006) identified a negativity bias that was also associated with smoking behaviour. Using a dotprobe detection task adapted for use in an fMRI scanner, Amin, Constable, and Canli (2004) identified an attentional bias that suggested that attention was diverted away from negative stimuli when they were presented alongside neutral words. No such pattern was identified when positive and 
neutral words were presented. One of the few studies in which positive and negative words were presented in tandem suggested that participants demonstrated a positivity bias in a dot-probe-type task (McCabe et al., 2000). In this study, the appearance of a negative stimulus with either a positive or a neutral stimulus increased response times to those stimuli. No significant effect was detected when neutral and positive words were presented, reinforcing the idea that these effects are due to an aversive response to negative stimuli, rather than to positive or neutral stimuli actively drawing attention. In the present study, participants were presented with multiple affective stimuli as part of a single image, extending the evidence presented here in dual-stimulus presentation research.

The design of the study made it possible to detect whether (a) the order in which objects changed within images affected response latencies independent of valence; (b) the valence of the preceding object change affected subsequent response latencies; and (c) the order in which entire image blocks were presented affected response latencies. Average completion times were also examined to assess the resource implications of a repeated measures design.

\section{Method}

\section{Participants}

A total of 97 undergraduate psychology participants (86 female, 11 male; $M=23.8$ years, $S D=6.41$ ) were recruited to take part in a study ostensibly examining perceptual processing. Participants with any form of photosensitive epilepsy were not allowed to participate due to the flickering nature of the images.

\section{Design}

This study used a $3 \times 2$ mixed factors design with Object Valence (positive, negative, or neutral) as a withinparticipants factor and Image Block Order (AB, BA) as a between-participants factor. The dependent variable was mean correct change detection latency. On each image, three objects were displayed (a positive, a negative, and a neutral object). Versions of each image appeared on five consecutive trials. Changes occurred to one of the three objects on separate trials, until all three objects had changed. On two of the five trials, no change occurred. These no-change trials were used to minimise participants' ability to employ a strategy to predict where changes might next appear within each image. Within each image, the layout of objects (left, centre, or right) was counterbalanced using a Latin square design. A Latin square design was also used to determine the order in which each object (positive, negative, or neutral) was changed (first, second, or last). A total of six different images were used throughout the study, generating a total of 30 trials across all six images. These images were grouped into two sets of three (Set A or Set $B$ ), allowing us to define two image block orders (AB, BA). While it would have been preferable to present the images in a fully Latin square design, the number of permutations made this unworkable, so two distinct orders were selected in order to make a basic comparison of the effect of image block order. Object layouts and change orders were counterbalanced across the two image blocks.

Stimuli and apparatus

Stimulus validation involved asking 12 independent judges to rate the valence of a corpus of 40 objects on a scale from 1 (extremely negative) to 7 (extremely positive). The six neutral objects were selected as those with mean ratings closest to 4, and the six positive and six negative objects were selected to have mean ratings closest to 7 and 1 , respectively. An analysis of valence ratings revealed a main effect of valence, $F(1,140)=74.02, p<.001, \eta_{\mathrm{p}}{ }^{2}=.514$, with positive object ratings $(M=5.70, S D=1.77)$ being significantly higher than both neutral $(M=3.54, S D=1.67)$ and negative $(M=2.78, S D=1.48)$ object ratings, $p \mathrm{~s}<$ .001 . Negative object ratings were also significantly lower than neutral ratings, $p<.05$.

Figure 1 illustrates a typical change trial: The originating stimulus was displayed for $250 \mathrm{~ms}$, followed by a mask of white noise for $80 \mathrm{~ms}$, the changed stimulus for a further $250 \mathrm{~ms}$, and finally another mask for $80 \mathrm{~ms}$. This sequence repeated until a response was given by the participant, or until a time limit of $30 \mathrm{~s}$ had elapsed. Images were presented at the centre of the screen, with the monitor resolution set to $800 \times 600$ pixels and images sized at $550 \times$ 400 pixels, such that they occupied approximately two thirds of the visible screen.

The experiment was run on a Windows-based PC, using a 15-in. CRT monitor. The design software package Macromedia Flash MX 7 was used to programme the paradigm. Responses were given using a standard twobutton PS/2 mouse device. Participants were seated approximately $50 \mathrm{~cm}$ from the screen, with their seat height was adjusted so that their eye level was perpendicular to the centre of the screen.

\section{Procedure}

Participants were asked to sit in front of a computer where instructions on how to proceed were given, and were randomly assigned to either the $\mathrm{AB}$ or the $\mathrm{BA}$ image block order condition by the computer programme. Included in 
Fig. 1 Example of a typical change trial and the associated timings

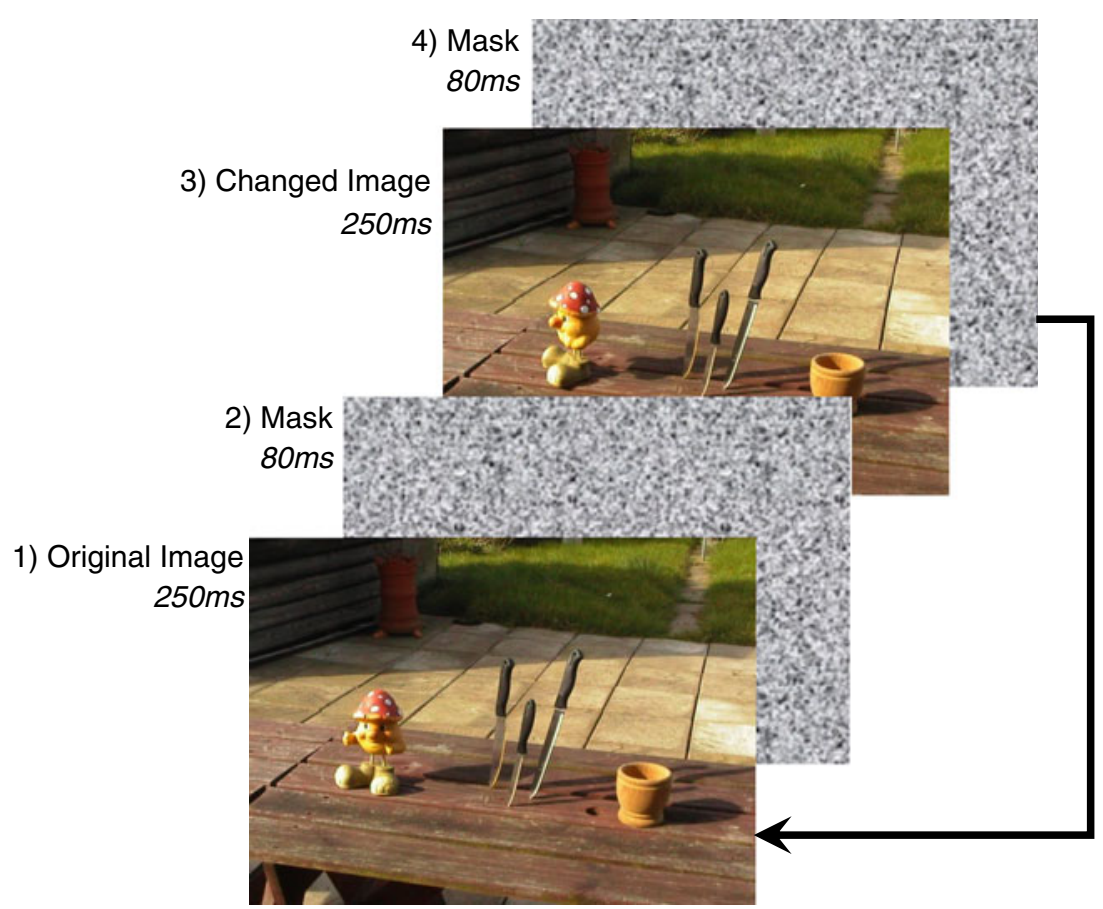

the instructions was a warning that they should discontinue the task if at any point they felt uncomfortable or experienced symptoms of nausea/dizziness whilst viewing the flickering images. No participants asked to be withdrawn or reported such symptoms. Participants were presented with images containing three objects of positive, neutral, or negative valence and used the mouse to give responses during each trial by clicking on an object if they saw it changing. Participants initiated trials at their own pace by clicking on a small green button located at the bottom centre of the computer screen. As well as allowing participants to take short breaks as they felt necessary, this also ensured that the mouse cursor was in the same position at the start of each trial.

\section{Results}

Task completion times

The maximum time a participant could take to complete the flicker task was $15 \mathrm{~min}$ - that would mean that no responses were given and that the 30-s time limit was allowed to elapse for all 30 trials. In practice, participants took an average of $7.59 \mathrm{~min}$ to complete the task $(S D=$ 0.92 , range $=4.84$ ).

\section{Data preparation}

Following previous studies (e.g., Schoenmakers, Wiers, Jones, Bruce, \& Jansen, 2007; Townshend \& Duka, 2004) utilising a variety of attentional bias measures, any responses below $200 \mathrm{~ms}$ from the initial presentation of a change (i.e., Step 3 in Fig. 1) were first coded as incorrect, because no change could have been detected before this point (N.B.: no response times actually fell below this limit). For each participant, responses falling above two standard deviations from their mean latency were also excluded from analysis as outliers. Erroneous responses (failing to respond on a change trial or clicking on the wrong object in the image) were also excluded. Any participants with more than $20 \%$ of their data missing after this were excluded from further analysis - for these reasons, 14 participants' data were subsequently excluded from the final analysis. It should be noted that all errors made were the result of participants failing to give a response when a change was occurring, and none were due to participants identifying a change in the wrong location. This high rate of attrition due to erroneous responding, over half of which occurred during the first two images (that is, within the first 10 trials), suggests that a practice block would be beneficial, to ensure that participants are able to fully understand the task requirements.

To correct for significant nonnormality in the response time data, a log transformation was applied. The untransformed means (in seconds) are presented throughout for ease of interpretation.

No-change trials were not subjected to inferential analysis, since many participants reported having given a response in these trials when they had not in fact seen a change occur, undermining the reliability of all data collected during these trials. The primary reason given for 
this was that participants felt that the 30 -s trial duration required in no-change trials was too long. Future implementations of this paradigm should therefore include an option for participants to actively indicate if they believe that no change is occurring within the image, although in cases where a no-change trial occurs after all three objects have changed already within an image, care should be taken to exclude such response latencies from analysis.

Image block order

A two-way ANOVA was conducted on the log-transformed response time data (in seconds), with Image Block Order as a between-participants factor and Object Valence as a withinparticipants factor. A significant main effect of object valence was revealed, $F(2,162)=21.17, p<.001, \eta_{\mathrm{p}}{ }^{2}=.21$. Planned contrasts revealed that response times to positive objects were significantly faster than those to both neutral and negative objects [Pos-Neu, $F(1,81)=42.51, p<.001, \eta_{\mathrm{p}}{ }^{2}=$ .34 ; Pos-Neg, $\left.F(1,81)=30.66, p<.001, \eta_{\mathrm{p}}^{2}=.28\right)$, which in turn were not significantly different from one another (Neu-Neg, $F(1,81)=0.161, p>.05, \eta_{\mathrm{p}}{ }^{2}=.002$; Pos, Neu, and Neg, $M \mathrm{~s}=3.401,4.450$, and 4.366; $S D \mathrm{~s}=1.32,1.87$, and 1.82 , respectively).

The main effect of image block order was not significant, $F$ $(1,81)=2.05, p>.05, \eta_{\mathrm{p}}{ }^{2}=.03$, although the Image Block Order $\times$ Object Valence interaction approached significance, $F(2,162)=2.81, p=.063, \eta_{\mathrm{p}}{ }^{2}=.034$. Figure 2 shows that across the two order conditions, positive response times were fastest, but the profile of neutral and negative latencies

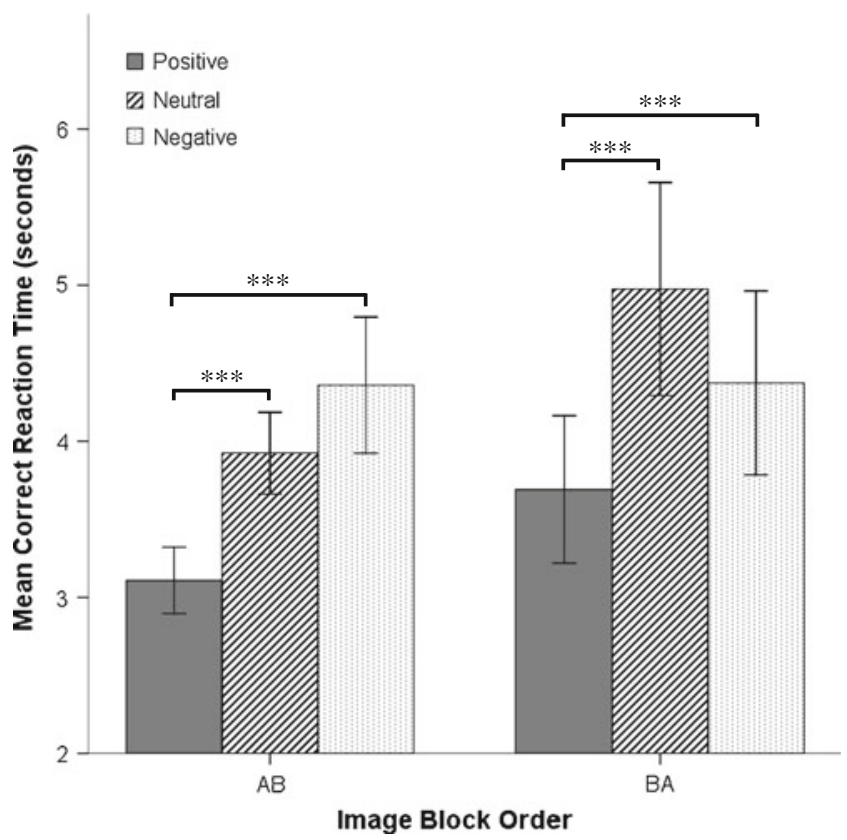

Fig. 2 Mean correct response latencies for positive, neutral, and negative changes for each image block order $(95 \%$ confidence intervals shown). ${ }^{* * *} p<.001$ varied, albeit nonsignificantly, between blocks. Therefore, while image block order did not seem to significantly affect the response latency profile to the objects within images, it nonetheless seems prudent to fully randomise blocks in future implementations of this paradigm to avoid any potentially confounding order effects.

Further exploratory analyses investigated whether the effects of object valence reported above were affected by the valence of the preceding object, by object position, and by change order.

Effect of preceding change valence on response latencies

As we used a multiple-trial implementation of the flicker paradigm, we explored whether any carryover effects occurred across trials. Specifically, we analysed whether the valence of the object that had changed in the preceding trial was positive, neutral, or negative, to see whether this affected response latencies on subsequent trials. A two-way ANOVA was conducted to examine the effects of the preceding change valence on response times, with Object Valence (positive, neutral, or negative) and Preceding Change Valence (positive, neutral, negative, or none ${ }^{1}$ ) as within-participants factors. The main effect of object valence, as previously identified, was significant, $F(2,164)=15.0, p<.001, \eta_{\mathrm{p}}^{2}=.155$. The main effect of preceding change valence was also significant, $F(3$, 246) $=13.46, p<.001, \eta_{\mathrm{p}}^{2}=.141$. Bonferroni comparisons showed that response latencies in the none condition $(M=4.66, S D=1.67)$ were significantly slower than those in the positive $(p<.02, M=3.99, S D=1.67)$, neutral $(p<.001, M=3.79, S D=1.48)$, and negative $(p<.001, M=$ $3.63, S D=1.48)$ preceding change conditions. Positive, neutral, and negative preceding change response latencies did not differ from one another significantly (all $p \mathrm{~s}>.8$ ). Most importantly, the Object Valence $\times$ Preceding Change Valence interaction was not significant, $F(3$, 246) $=0.469, p>.7, \eta_{\mathrm{p}}^{2}=.006$. In other words, participants were slower to respond to the first change within each image block, but the valence of the preceding change had no effect on change detection latencies.

Effect of preceding change presence on response latencies

In an analysis related to the previous one assessing whether the valence of the preceding change affected response latencies, a two-way ANOVA was carried out to identify whether the simple presence or absence of a change in the previous trial affected response latencies to each object type, with Preceding Change Presence (preceding change, no preceding change) and Object Valence (positive, neutral,

\footnotetext{
${ }^{1}$ None refers to change trials at the start of each image block, which did not have any change preceding them.
} 
or negative) as within-participants factors. The main effect of object valence remained significant, $F(2,164)=$ 27.07, $p<.001, \eta_{\mathrm{p}}{ }^{2}=.248$. The main effect of preceding change presence was not significant, $F(1,164)=0.569, p>$ $.05, \eta_{\mathrm{p}}{ }^{2}=.007$. Most importantly, the Object Valence $\times$ Preceding Change Valence interaction was not significant, $F$ $(2,164)=2.61, p>.05, \eta_{\mathrm{p}}{ }^{2}=.031$, demonstrating that the location of no-change trials did not have an impact on the overall profile of response latencies to each object type.

\section{Object position}

A two-way ANOVA was carried out to detect any effects of object position on response times to change detection, with Object Position (left, centre, or right) and Object Valence (positive, neutral, or negative) as within-participants factors. A nonsignificant trend was detected for object position, $F(2,328)=2.913, p=.057, \eta_{\mathrm{p}}{ }^{2}=.034$, such that images presented on the left of the screen were associated with slightly slower response latencies $(M=$ $4.28, S D=1.55)$ than were objects presented in the centre $(M=4.01, S D=1.36)$ or the right $(M=3.98, S D=1.39)$ of the images. Post hoc comparisons were all nonsignificant ( $p$ s $>.1$ ). The significant main effect of object valence remained significant in this analysis, as previously described, $F(2,328)=22.97, p<.001, \eta_{\mathrm{p}}{ }^{2}=.22$. Unexpectedly, a significant Object Valence $\times$ Object Position interaction was identified, $F(4,328)=12.01, p<$ $.001, \eta_{\mathrm{p}}{ }^{2}=.13$. Simple main effects comparisons for this interaction revealed that there was no significant difference between response latencies for any objects presented in the left side of the image ( $p s>.05)$, while the profile of response latencies (i.e., positive $<$ neutral $=$ negative) identified previously was consistent for objects presented in both the centre and right positions. In the absence of additional data such as handedness, it is not possible to speculate further on the reasons for this lack of effect in the left visual field. However, we suggest that this confirms the necessity for effective counterbalancing of objects within images, to ensure that systematic errors are not introduced into the data.

\section{Change order}

A two-way ANOVA was conducted to test for any effects of change order on change detection latencies, with Change Order (first, second, or last) and Object Valence (positive, neutral, or negative) as within-participants factors. A significant main effect of change order was revealed, $F(2$, $328)=39.13, p<.001, \eta_{\mathrm{p}}{ }^{2}=.32$. Post hoc analysis of the means, using Bonferroni correction for multiple comparisons, revealed that the change detection latencies for the first change $(M=4.66, S D=1.6)$ were significantly slower than those for both the second $(M=4.13, S D=1.56, p<.01)$ and last $(M=$ $3.48, S D=1.52, p<.001)$ changes, which in turn were significantly different from one another $(p<.001)$. Again, a significant main effect of object valence was revealed, $F(2$, $328)=19.5, p<.001, \eta_{\mathrm{p}}{ }^{2}=.19$, with positive objects being responded to more quickly than neutral $(p<.001)$ and negative $(p<.001)$ objects, which did not differ from one another $(p>.05)$.

The Object Valence $\times$ Change Order interaction was found to be significant, $F(2,328)=5.643, p<.05, \eta_{\mathrm{p}}{ }^{2}=$ .064 (Fig. 3). A simple main effects analysis was conducted to clarify this interaction. There was no significant main effect of object valence at the last level of change order, $F$ $(2,164)=1.24, p>.05, \eta_{\mathrm{p}}{ }^{2}=.015$. For both the first, $F(2$, $164)=10.16, p<.01, \eta_{\mathrm{p}}{ }^{2}=.11$, and second, $F(2,164)=$ $23.15, p<.001, \eta_{\mathrm{p}}{ }^{2}=.22$, levels of change order, there was a significant main effect of object valence. Bonferroni comparisons revealed that at both of these levels, change detection latencies were fastest for positive object changes (all $p \mathrm{~s}<.001$ ), whilst neutral and negative change detection latencies were not significantly different (all $p \mathrm{~s}>.05$ ). As a result, we recommend that the final change within each image block be excluded from analyses.

\section{Discussion}

This article reports the development and implementation of a modified flicker paradigm to examine attentional biases in human information processing. Specifically, we developed a repeated measures format with nonverbal responses to investigate response biases as a function of repeated trials, across multiple trial types, for emotionally laden objects. Methodologically, our findings lead to four recommendations for using this repeated measures flicker paradigm. First, a practice block should be included, to allow participants to orient themselves with the task requirements. Second, the order of image blocks should be randomised across participants, to minimise any confounding effects of image block order. Third, only the first and second changes within each image should be considered critical trials. Given that by the third change participants would be able to strategically ignore the two objects that have already changed, the response times to the final change within each image are clearly unreliable indicators of attentional bias. However, the consistent demonstration of a positivity bias, irrespective of the valence of the preceding changes, suggests that carryover effects did not occur in this repeated measures implementation of the flicker paradigm. Finally, the ability to give a response on no-change trials should be included, to avoid participants giving erroneous responses in these trials to "hurry up" the task. Aside from these modifications, the repeated measures flicker paradigm does 
Fig. 3 Mean correct response latencies for positive, neutral, and negative changes at each change order, irrespective of change valence $(95 \%$ confidence intervals shown). ${ }^{* *} p<.01$

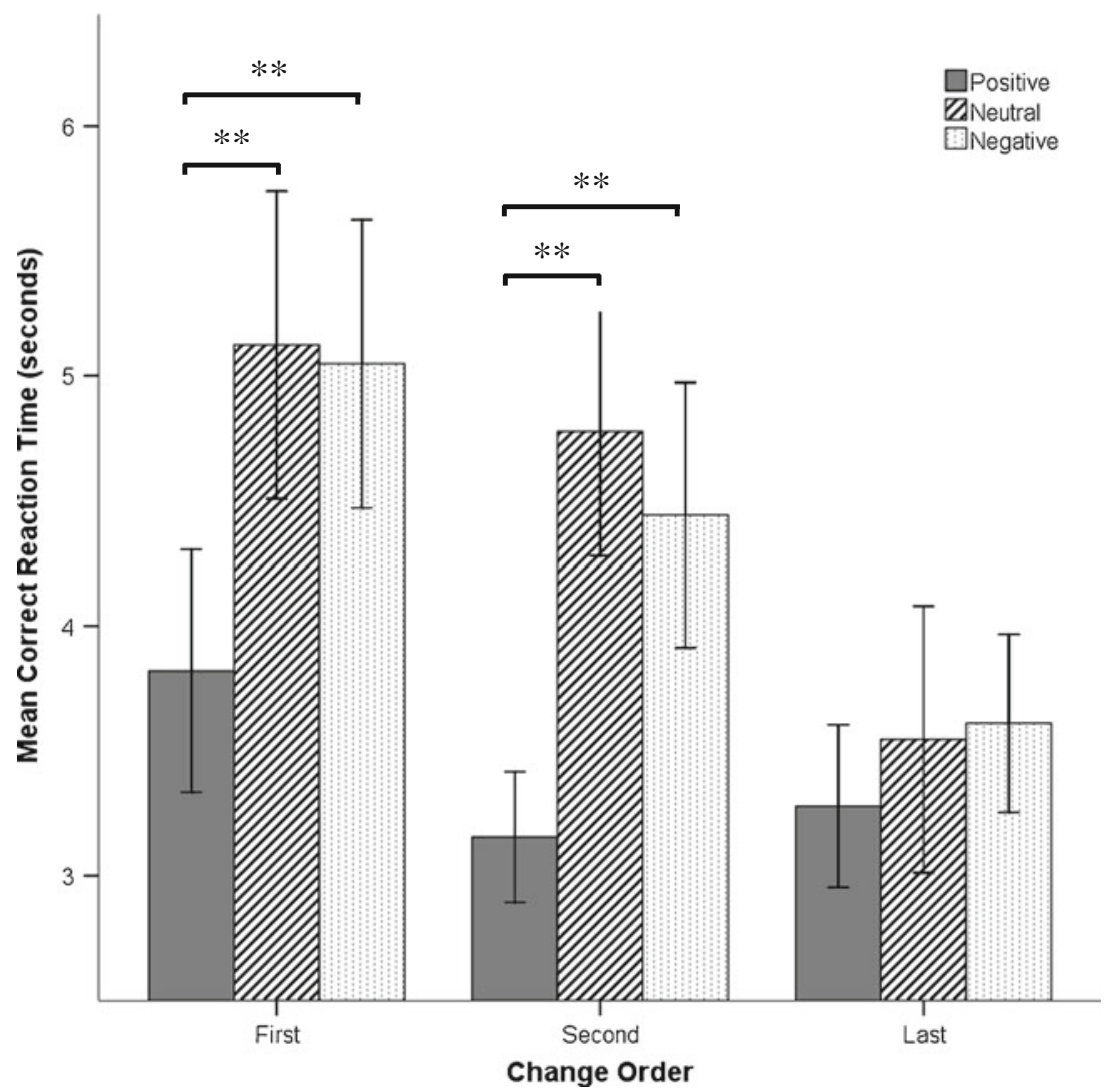

seem robust, and we did not find evidence of carryover or priming effects as a result of multiple stimulus presentations between the first and second change trials. As such, our findings suggest that concerns over the utility of this paradigm when used with a repeated measure are unwarranted, and it is indeed possible to obtain reliable data related to attentional biases for concern-related stimuli. This paradigm will therefore allow us to explore attentional biases towards multiple stimulus types within individuals, while also having the advantage over other types of attentional bias measure that objects may be presented within a coherent, realistic image. Future research should focus on the application of this procedure to other stimulus types, across a broader range of participant groups.

In addition to methodological implications, this experiment has also revealed an interesting finding, in light of past literature assessing attentional biases for affective stimuli. Our findings suggest the existence of a positivity bias, such that participants responded more quickly to changes to positive objects; this effect was consistent across all analyses when we took into account change order, preceding change valence and presence, object position, and image block order. There are three possible interpretations of this finding. First, one might suggest that participants simply had an attentional bias towards positive stimuli, hence the faster response latencies and the lack of a difference between neutral and negative change detection latencies (Hypothesis 1). Second, it may be that negative objects grab attention at a very early stage, and due to their aversive nature, attention was subsequently diverted towards more positive stimuli (Hypothesis 2). This might well occur within the first 250 -ms originating stimulus presentation. Indeed, it has been shown that the valence of stimuli is evaluated rapidly in studies of inattentional blindness (e.g., Mack \& Rock, 1998), that negative stimuli can be evaluated within 14 ms (Dijksterhuis \& Aarts, 2003), and that eye saccades could have been initiated and completed well before the first change occurred (i.e., within the first $330 \mathrm{~ms}$ of each trial; Rayner, 1998). Finally, it could be that negative stimuli do receive more attention than other stimuli (or indeed an equal amount), but that our participants were simply unable to disengage from them in order to initiate a timely response once a change had been detected (Hypothesis 3). Hypothesis 3 is problematic for two reasons. First, it is not clear why neutral latencies would be no different from negative latencies if attention was being diverted from the negative objects. Second, positive latencies were quicker by far than latencies to both neutral and negative objects, and it is not clear why this should be the case if positive images are not themselves attracting attention.

Hypothesis 2, that negative objects are evaluated as being negative at a preconscious level, has more appeal. It would then follow that participants would spend less time 
searching the less pleasant area of the image, leading to decreased response latencies in negative change trials. However, if one posits that negative stimuli deflected attention due to their unpleasant nature, it would stand to reason that (a) neutral stimuli should be responded to more quickly than negative stimuli, and (b) positive stimuli should not ipso facto be responded to more quickly that neutral stimuli. On this basis, we suggest that any account of these findings must suppose that positive stimuli actively drew attention (Hypothesis 1). This does not imply that negative stimuli do not generate a complementary aversive response, but a true positivity bias must exist in any case.

\section{Conclusion}

The flicker paradigm is a welcome addition to the battery of tasks available to researchers who are interested in assessing attentional biases in a variety of domains. However, in modifying the version of the paradigm developed by Rensink et al. (1997), a number of key features were lost, particularly the inclusion of multiple trials. To an extent this was understandable, because repeated exposure to stimuli related to behaviours such as drug or alcohol use may well lead to unwanted priming effects. One of our aims in the present study was to develop a version of the flicker task that retained many of the features that visual perception researchers would be familiar with, to see whether such a version could reliably be used to assess attentional biases. Our findings to this extent have been encouraging: Although one might take further steps in developing this paradigm (e.g., using more complex visual stimuli or even moving images), concerns over the reliability of the task, particularly when utilised in its full form, have not been shown to be justified.

Author Note We are grateful to Nicholas Moss for technical assistance in developing this paradigm, and to Tania Peart for her assistance in the collection of data for this project.

\section{References}

Amin, Z., Constable, T., \& Canli, T. (2004). Attentional bias for valenced stimuli as a function of personality in the dot-probe task. Journal of Research in Personality, 38, 15-23.

Beare, L., Sturt, P., Bruce, G., \& Jones, B. T. (2007). Heroin-related attentional bias and monthly frequency of heroin use are positively associated in attenders of a harm reduction service. Addictive Behaviors, 32, 784-792.

Dijksterhuis, A., \& Aarts, H. (2003). On wildebeests and humans: The preferential detections of negative stimuli. Psychological Science, $14,14-18$
Drobes, D. J., Elibero, A., \& Evans, D. E. (2006). Attentional bias for smoking and affective stimuli: A Stroop task study. Psychology of Addictive Behaviors, 20, 490-495.

Ebbinghaus, H. (1913). Memory: A contribution to experimental psychology (Trans. H. A. Ruger \& C. E. Bussenius). New York: Columbia University, Teachers College (Original work published in German in 1885).

Fox, E., Russo, R., \& Dutton, K. (2002). Attentional bias for threat: Evidence for delayed disengagement from emotional faces. Cognition and Emotion, 16, 355-379.

Ito, T. A., Larsen, J. T., Smith, N. K., \& Cacioppo, J. T. (1998). Negative information weighs more heavily on the brain: The negativity bias in evaluative categorizations. Journal of Personality and Social Psychology, 75, 887-900.

Jones, B. T., Bruce, G., Livingstone, S., \& Reed, E. (2006). Alcoholrelated attentional bias in problem drinkers with the flicker change blindness paradigm. Psychology of Addictive Behaviors, 20, 171-177.

Jones, B. C., Jones, B. T., Blundell, L., \& Bruce, G. (2002). Social users of alcohol and cannabis who detect substance-related changes in a change blindness paradigm report higher levels of use than those detecting substance-neutral changes. Psychopharmacology, 165, 93-96.

Jones, B. T., Jones, B. C., Smith, H., \& Copley, N. (2003). A flicker paradigm for inducing change blindness reveals alcohol and cannabis information processing biases in social users. Addiction, 98, 235-244.

Jones, B. T., Macphee, L. M., Broomfield, N. M., Jones, B. C., \& Espie, C. A. (2005). Sleep-related attentional bias in good, moderate, and poor (primary insomnia) sleepers. Journal of Abnormal Psychology, 114, 249-258.

Mack, A., \& Rock, I. (1998). Inattentional blindness. Cambridge, MA: MIT Press.

McCabe, S. B., Gotlib, I. H., \& Martin, R. A. (2000). Cognitive vulnerability for depression: Deployment of attention as a function of history of depression and current mood state. Cognitive Therapy and Research, 24, 427-444.

Rayner, K. (1998). Eye movements in reading and information processing: 20 years of research. Psychological Bulletin, 124, 372-422.

Rensink, R. A. (2002). Change detection. Annual Review of Psychology, 53, 245-277.

Rensink, R. A., O’Regan, J. K., \& Clark, J. J. (1997). To see or not to see: The need for attention to perceive changes in scenes. Psychological Science, 8, 368-373.

Schoenmakers, T., Wiers, R. W., Jones, B. T., Bruce, G., \& Jansen, A. T. M. (2007). Attentional re-training decreases attentional bias in heavy drinkers without generalization. Addiction, 102, 399-405.

Segerstrom, S. C. (2001). Optimism and attentional bias for negative and positive stimuli. Personality and Social Psychology Bulletin, 27, 1334-1343.

Simons, D. J., \& Chabris, C. F. (1999). Gorillas in our midst: Sustained inattentional blindness for dynamic events. Perception, 28, 1059-1074.

Simons, D. J., \& Rensink, R. A. (2005). Change blindness: Past, present and future. Trends in Cognitive Sciences, 9, 16-20.

Townshend, J. M., \& Duka, T. (2004). Attentional bias associated with alcohol cues: Differences between heavy and occasional social drinkers. Psychopharmacology, 157, 67-74.

Werner, S., \& Thies, B. (2000). Is "change blindness" attenuated by domain-specific expertise? An expert-novices comparison of change detection in football images. Visual Cognition, 7, 163-173. 